\title{
High Field Side Lower Hybrid Current Drive Simulations for Off- axis Current Drive in DIII-D
}

\author{
S.J. Wukitch ${ }^{1, *}$, S. Shiraiwa ${ }^{1}$, G.M. Wallace ${ }^{1}$, P.T. Bonoli ${ }^{1}$, C. Holcomb ${ }^{2}$, and R.I. Pinsker ${ }^{3}$ \\ ${ }^{1}$ Massachusetts Institute of Technology, Plasma Science and Fusion Center, Cambridge, Massachusetts 02139, USA \\ ${ }^{2}$ Lawrence Livermore National Laboratory, Livermore, California 94551, USA \\ ${ }^{3}$ General Atomics, P.O. Box 85608, San Diego, California 92186-5608, USA
}

\begin{abstract}
Efficient off-axis current drive scalable to reactors is a key enabling technology for developing economical, steady state tokamak. Previous studies have focussed on high field side (HFS) launch of lower hybrid current drive (LHCD) in double null configurations in reactor grade plasmas and found improved wave penetration and high current drive efficiency with driven current profile peaked near a normalized radius, $\rho$, of $0.6-0.8$, consistent with advanced tokamak scenarios. Further, HFS launch potentially mitigates plasma material interaction and coupling issues. For this work, we sought credible HFS LHCD scenario for DIII-D advanced tokamak discharges through utilizing advanced ray tracing and Fokker Planck simulation tools (GENRAY+CQL3D) constrained by experimental considerations. For a model and existing discharge, HFS LHCD scenarios with excellent wave penetration and current drive were identified. The LHCD is peaked off axis, $\rho \sim 0.6-0.8$, with FWHM $\Delta \rho=0.2$ and driven current up to $0.37 \mathrm{MA} / \mathrm{MW}$ coupled. For HFS near mid plane launch, wave penetration is excellent and have access to single pass absorption scenarios for variety of plasmas for $n_{\|}=2.6-3.4$. These DIII-D discharge simulations indicate that HFS LHCD has potential to demonstrate efficient off axis current drive and current profile control in DIII-D existing and model discharge.
\end{abstract}

\section{Introduction}

Efficient off-axis current drive (OACD) scalable to reactors is a key enabling technology for economical, steady state tokamak. Efficient OACD scalable to reactors is yet to be demonstrated both from efficiency and survivability perspective. Studies of reactor grade, double null plasmas utilizing high field side (HFS) launch lower hybrid current drive (LHCD) have found improved wave penetration enabling efficient OACD.[1] Further, HFS launch potentially mitigates plasma material interaction and coupling issues due to quiescent HFS scrape off layer (SOL)[2], lower plasma particle[3] and neutron flux, and improved impurity screening[4]. HFS LHCD in DIII-D discharges offer an opportunity to validate HFS wave physics and SOL benefits for RF actuators.

In addition to primarily RF constraints, the analysis presented here optimizes for scenarios compatible with DIII-D advanced tokamak and scenario development research.[5] A key ingredient is current profile optimization.[6] Given the existing and planned DIII-D off axis current drive actuators (neutral beam[7], electron cyclotron[8], and helicon[9]), the HFS LHCD is to drive current at normalized minor radius, $\rho$, in the range of 0.6-0.8 with current density approaching $0.4 \mathrm{MA} / \mathrm{m}^{2}$ for fields down to $1.6 \mathrm{~T}$ with central densities approaching
$1 \times 10^{20} \mathrm{~m}^{-3}$. The goal of this work is to identify a credible HFS LHCD scenario for DIII-D. The simulation tool utilized in this study is GENRAY+CQL3D (advanced ray tracing and Fokker Planck solver[10,11]) for which existing and model DIII$\mathrm{D}$ discharges were investigated.

The motivation to investigate HFS LHCD in DIII-D derives from the possibility to significantly improve the wave penetration and absorption compared to low field side (LFS) launch. For lower hybrid (LH) waves, wave access is a set by wave accessibility[12] and the condition for electron Landau damping[13]. For a given wave frequency, the minimum index of refraction, $\mathrm{n}_{\|}$, that will penetrate into plasma is proportional to $n_{e}^{1 / 2} / B$ where $n_{e}$ is plasma density and $\mathrm{B}$ is magnetic field. Ideally, the wave penetrates into the plasma until electron Landau damped which can be expressed as $\mathrm{n}_{\|} \geq\left(30 / T_{\mathrm{e}}\right)^{1 / 2}$ where $\mathrm{T}_{\mathrm{e}}$ is electron temperature in $\mathrm{keV}[14]$. On the HFS, the toroidal field is higher and allows launch of lower $n_{\|}$ waves that penetrate farther into the plasma core before damping. A further benefit to lower $\mathrm{n}_{\|}$launch is that the waves are absorbed at higher $\mathrm{T}_{\mathrm{e}}$ yielding a higher current drive efficiency, $\sim 1 / \mathrm{n}_{\|}^{2}$, because wave momentum is transferred to less collisional electrons.[15] Looking towards reactor concepts where current drive efficiency is the primary parameter to be optimized, the poloidal position is selected to balance the effects of toroidicity

Corresponding author: wukitch@psfc.mit.edu 


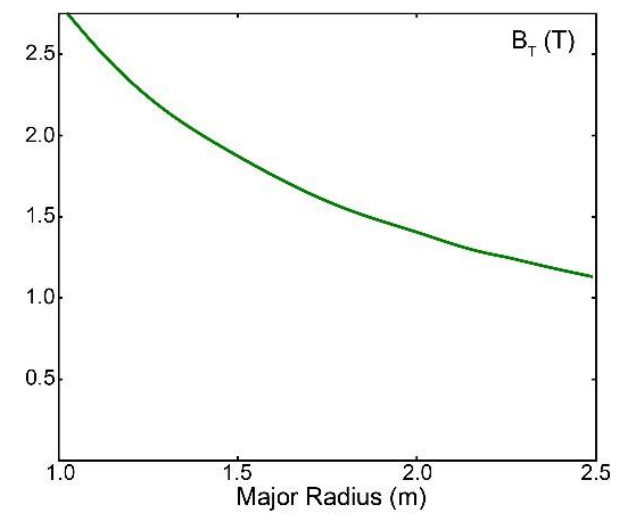

Fig. 1 Magnetic field profile for $147634(1.66 \mathrm{~T}$ discharge) shows that the HFS local magnetic field is $2.5 \mathrm{x}$ the LFS value.

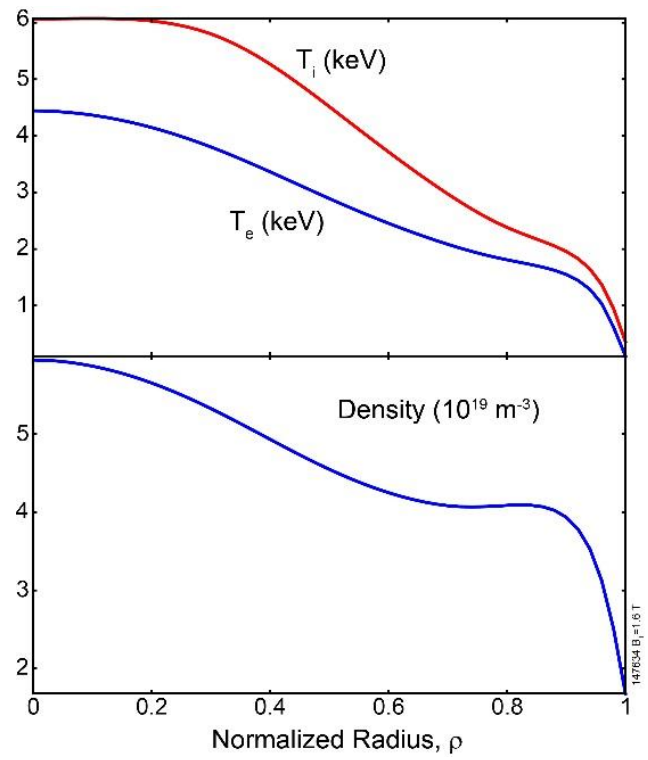

Fig. 2 Electron density, ion and electron temperature profiles are peaked.

and poloidal field to maintain the smallest $\mathrm{n}_{\|}$possible. In DIII-D, the primary consideration is the damping location. Thus, proper poloidal positioning allows for waves to penetrate and damp in region $\rho \sim 0.6-0.8$.

\section{HFS LHCD Scenario Identification}

As mentioned above, LFS LHCD is unattractive in DIII$\mathrm{D}$ and reactor plasmas due to wave penetration into the plasma core.[1] Using a typical low $\mathrm{q}_{\min }(\mathrm{q}$ is safety factor) discharge (147634), the mid plane B-field, density electron and ion temperature profiles are shown in figure 1 and 2. The waves launched with $\mathrm{n}_{\|}=2.7$ at 4.6 $\mathrm{GHz}$ from the outboard mid plane fail to penetrate into the plasma core and pass through the divertor region as shown in figure 3 . The discharge profiles are representative of profiles in other DIII-D advanced tokamak (AT) and model discharges investigated here. The study examined a variety of discharges with different launcher locations to identify launch positions to optimize wave penetration, absorption location, and current drive efficiency. The waves were launched assuming four poloidal locations to represent an

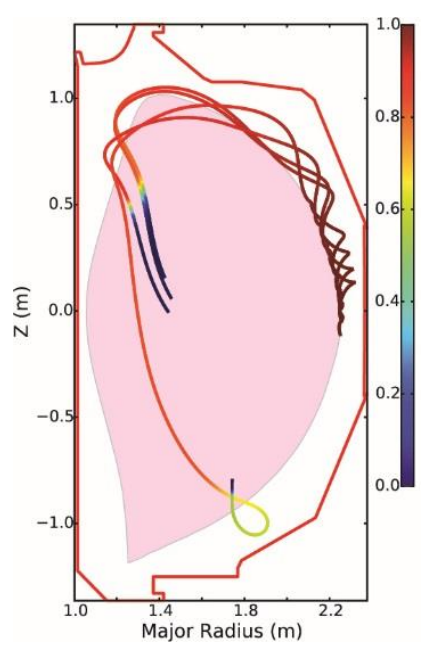

Fig. 3 LH waves launched from LFS mid plane remain in the plasma periphery for 147634 due to the density and low local B field.

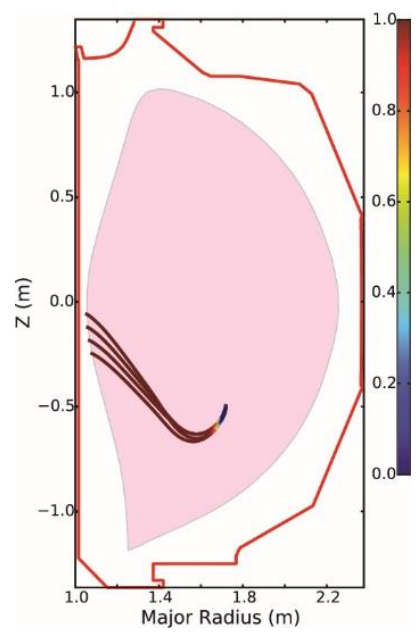

Fig. 4 LH waves launched from HFS near mid plane penetrate and single pass damp near $\rho \sim 0.6$ for 147634 .

expected coupler with a spectrum peaked near 2.7 and width of $+/-0.2$ which is consistent with 32 toroidal apertures. Two HFS launch positions showed the greatest promise: near the upper divertor and near the mid plane.

An example of near mid plane HFS launch position is shown in figure 4 . The rays are launched at poloidal positions 3-24 $\mathrm{cm}$ below the mid plane. The launch spectrum has a peak at $\mathrm{n}_{\|}=2.7+/-0.2$. The rays penetrate and drive $\sim 150 \mathrm{kA} / \mathrm{MW}$ peaked near $\rho \sim 0.6$ with a corresponding efficiency $0.12 \times 10^{20} \mathrm{~A}^{*} \mathrm{~W}^{-1} \mathrm{~m}^{-2}$. As shown in Figure 5, the simulation suggests with $1 \mathrm{MW}$ of coupled power can satisfy the required OACD in the range of $\rho \sim 0.6-0.8$ with current density approaching 0.4 $\mathrm{MA} / \mathrm{m}^{2}$ for AT discharges. The ray penetration and trajectory are sensitive to $\mathrm{n}_{\|}$and poloidal launch position. The rays from $-3 \mathrm{~cm}$ propagate to the largest $Z$ values with a few rays, $\mathrm{n}_{\|} \sim 2.5$, refracting back to the plasma edge at the top of the device. Launching the rays at or above the mid plane results in many more rays becoming trapped in the plasma edge.

An example of near upper divertor HFS launch position is shown in figure 6 . The rays are launched at poloidal 


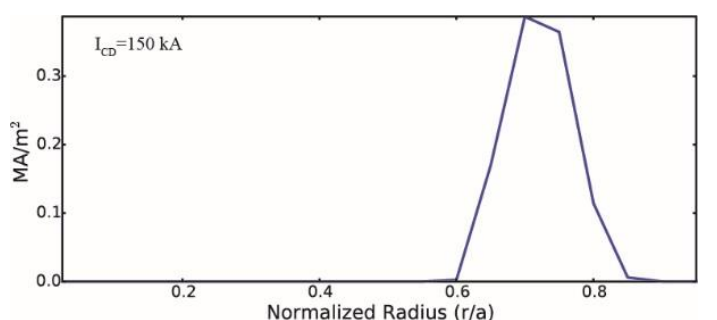

Fig. 5 Driven current profile for 147634 for near mid plane launch is peaked near $\rho \sim 0.6$ and approaching the required $0.4 \mathrm{MA} / \mathrm{m}^{2}$ with $1 \mathrm{MW}$ coupled.

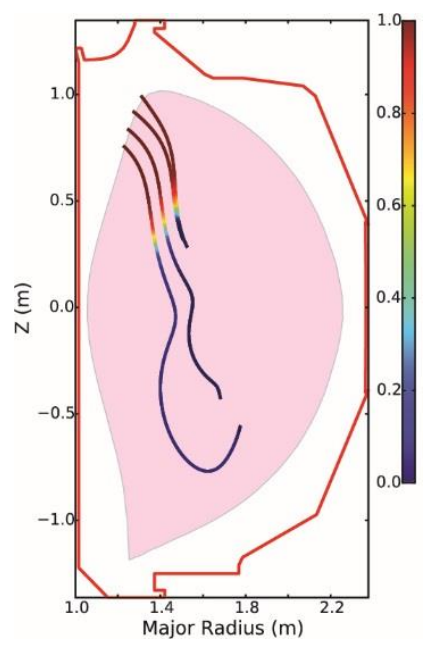

Fig. 6 Ray propagation for near upper divertor LH wave launch shows good wave penetration and absorption.

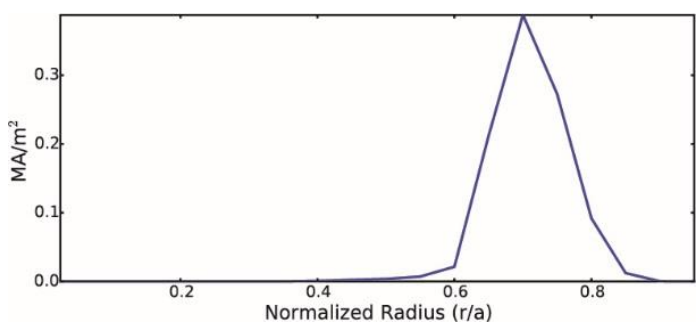

Fig. 7 Driven current profile is peaked near $\rho \sim 0.7$ with $\sim 140 \mathrm{kA}$ driven current.

positions 0.85 to $1.0 \mathrm{~m}$ above the mid plane. The launch spectrum is peaked at $n_{\|}=2.7+/-0.2$. The rays penetrate and damp near $\rho \sim 0.7$ and drive $\sim 140 \mathrm{kA}$ per MW coupled peaked with a corresponding efficiency $0.1 \times 10^{20}$ $\mathrm{A}^{*} \mathrm{~W}^{-1} \mathrm{~m}^{-2}$ as shown in Fig. 7. Moving the launch location to closer to the mid plane results in decreased penetration and multiple reflections. Although similar to the near mid plane launch in penetration and efficiency, the near upper divertor launch has practical considerations that make it less ideal. For upper discharges with upper X-points, the launcher would be in a zone of high heat flux and high density. The former would make a structure difficult to design and the latter may prevent the waves from penetrating into the plasma. Further, long distance coupling would be very challenging. The anticipated long distance coupling, the distance to the last closed flux surface (LCFS), is $>10$ $\mathrm{cm}$.

For near mid plane launch, the sensitivity to magnetic field, electron temperature, and density were examined. The general trend with $\mathrm{B}$-field is $\mathrm{n}_{\|}$required for damping at $\rho \sim 0.6-0.8$ increased to 3 as B-field decreased to $1.6 \mathrm{~T}$.

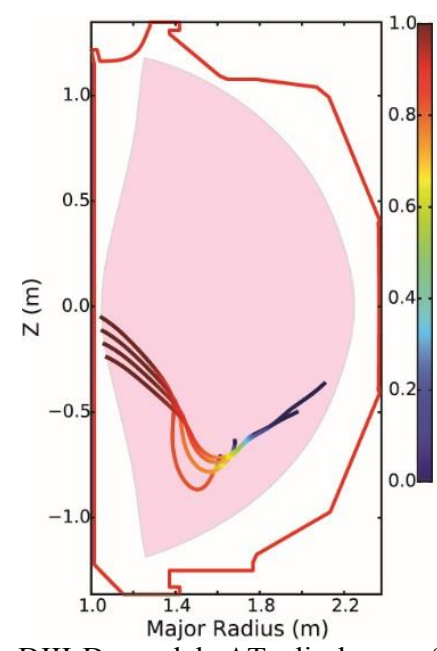

Fig. 8 For DIII-D model AT discharge (1.6 T), HFS launched near mid plane penetrate and has single pass damping.

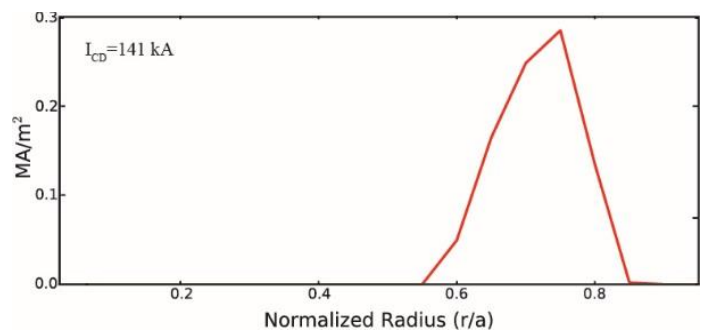

Fig. 9 Driven current profile for 1.6 T, DIII-D model AT discharge is peaked near $\rho \sim 0.7$ for near mid plane launch.

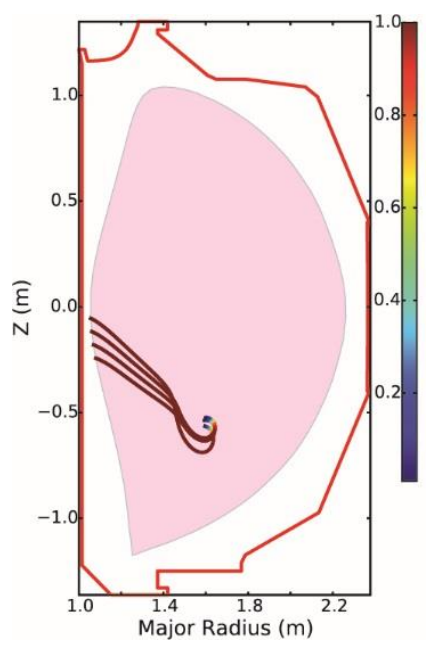

Fig. 10 For $144476(2$ T), HFS launch has excellent wave penetration and single pass damp near $\mathrm{r} \sim 0.6$.

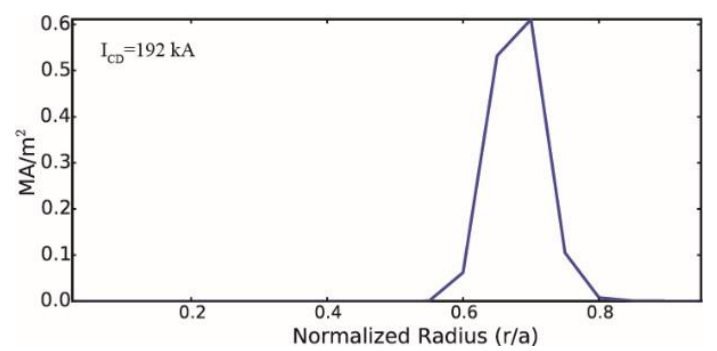

Fig. 11 Driven current profile for 144476 (2 T) DIII-D AT discharge for $n \|=2.6+/-0.2$ is peaked near $\mathrm{r} \sim 0.7$ and drives $\sim 190 \mathrm{kA} / \mathrm{MW}$ with current density $>0.4 \mathrm{MA} / \mathrm{m}^{2}$.

Fig 8 and 9 show the wave propagation and driven current for a DIII-D 1.6 T AT discharge. For $\mathrm{n}_{\|}=3+/-0.2$, 


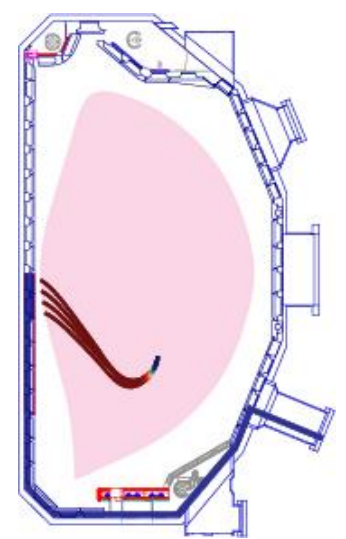

Fig. 12 The wave guide route fits within the invessel profile except on the inner wall and baffle plate.

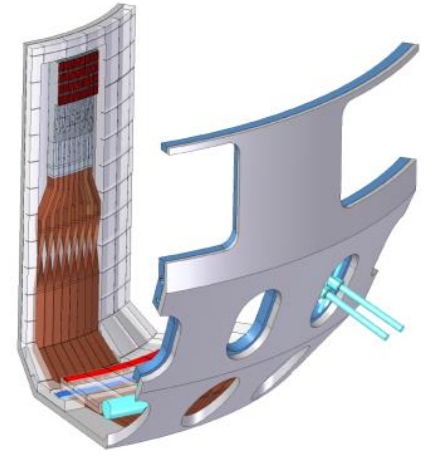

Fig. 13 Placement of HFS LHCD coupler and wave guide network in the DIII-D model vacuum vessel is challenging but appears plausible assuming one can increase the radius of the center column tiles $2.5 \mathrm{~cm}$.

the waves propagate and drive $\sim 150 \mathrm{kA} / \mathrm{MW}$ current between $\rho \sim 0.6-0.8$. To obtain $0.4 \mathrm{MA} / \mathrm{m}^{2}$ for this discharge, the HFS LHCD power would need to couple $\sim 1.3 \mathrm{MW}$. At $\sim 2 \mathrm{~T}$, the higher field allows a lower $\mathrm{n}_{\|}$to be launched and the corresponding driven current increases to $190 \mathrm{kA} / \mathrm{MW}$. At this level, the current density is sufficient to allow current profile control from a simulation perspective. Similar studies were done for density and temperature. As expected increasing the density required increasing $\mathrm{n}_{\|}$to 3.4 for central density $\sim 9 \times 10^{20} \mathrm{~m}^{-3}$ at $1.6 \mathrm{~T}$ and the driven current falls to $\sim 85$ $\mathrm{kA} / \mathrm{MW}$. At higher B-fields, the density window and driven current levels improve. For temperature, the primary impact was on single pass damping. Decreasing the temperature by $25 \%$ from existing discharges resulted in waves having multiple reflections where $\sim 85 \%$ of the power is damped on the first pass.

\section{Practical Outlook for HFS LHCD at DIII- D}

The conceptual launcher and wave guide network design is based on existing technology and is shown in Fig 12 and Fig 13. One WR-187 wave guide from a $250 \mathrm{~kW}$ klystron will be split into 16 apertures using two bijunctions[16] toroidally and a 4 way poloidal

\section{References}

[1]P.T. Bonoli et al., "Novel Reactor Relevant RF Actuator Schemes for the Lower Hybrid and the Ion Cyclotron Range of Frequencies". Preprint: 2016 IAEA Fusion Energy Conference, Kyoto [TH/5-1].

[2] N. Smick, B. Labombard, and I.H. Hutchinson, Nuclear Fusion 53, 023001 (2013).

[3] B. LaBombard, J.E. Rice, A.E. Hubbard, J.W. Hughes et al., Nuclear Fusion 44, 1047 (2004)

[4] B. Labombard,A. Kuang, D. Brunner, I. Faust et al., Nuclear Fusion 57, -76021 (2017)

[5] T. C. Luce, Fusion Sci. Tech. 48, 1212-1225 (2005).

[6] C. T. Holcomb, J. R. Ferron, T. C. Luce, T. W. Petrie, et al. Nuclear Fusion 54, 093009 (2014).

[7] J. M. Park, M. Murakami, C. C. Petty, W. W. Heidbrink et al., Phys. Plasmas 16, 092508 (2009).

splitter[17]. The conceptual launcher is eight WR-187 split into 32 toroidal apertures. This results in an expected power density of $<30 \mathrm{MW} / \mathrm{m}^{2}$ assuming 200 $\mathrm{kW}$ delivered and similar to power densities obtained in Tore Supra and planned for ITER.[16] The aperture dimension is $6 \mathrm{~cm}$ tall and $0.5 \mathrm{~cm}$ wide with a $1 \mathrm{~mm}$ septa and results in a peak $\mathrm{n}_{\|}=2.7$ for $\Delta \phi=90^{\circ}$ excitation. With the passive elements, the directivity is $\sim 62 \%$ and can access $2.4 \leq \mathrm{n}_{\|} \leq 3$ with $>55 \%$ directivity. The wave guide enters from the LFS, passes under the cryopump and divertor and up the inner wall with the long dimension along the vessel. Above the inner strike point, the wave guide twists to orient the wave guide's long dimension perpendicular to the inner column. To accommodate the twist, the inner column radius would need to increase by $2.54 \mathrm{~cm}$. For the AT plasmas simulated here, the smallest inner gap is $3.8 \mathrm{~cm}$ suggesting there is may be room to expand the inner column without impacting the target AT plasmas. In addition to the inner column tile modifications, a number of protective tiles and baffle plate would need to be modified to allow routing of the wave guides to the launcher. From an operational perspective, impurities from a metal surface on the inner wall needs to be assessed.

\section{Summary of HFS LHCD at DIII-D}

The simulations suggest efficient HFS LHCD off-axis current drive is possible for DIII-D existing and target AT discharges. An experiment based on these simulations could provide an opportunity to demonstrate $\mathrm{q}$ profile control for $\mathrm{B}_{\mathrm{T}} \geq 1.6 \mathrm{~T}, \mathrm{n}_{\mathrm{e}}<9 \times 10^{19} \mathrm{~m}^{-3}$ with high current drive efficiency and deposition peaked between $\rho=0.6-0.8$ compatible with high temperature plasmas and scalable to reactors. Further, such an experiment would allow investigation of HFS wave propagation and SOL benefits for RF actuators. The primary in-vessel challenge is increase of the central column radius.

Work supported by the U.S. Department of Energy, Office of Science, Office of Fusion Energy Sciences, using User Facility DIII-D, under Award Number DE-FC02-04ER54698 and by US DoE Contract No. DE-FC02-01ER54648 under a Scientific Discovery through Advanced Computing Initiative.

[8] R. Prater and C. C. Petty, Fusion Sci. Tech. 48, 1141-1148 (2005).

[9] R. Prater, C. P. Moeller, R. I. Pinsker, M. Porkolab et al., Nucl. Fusion 54, 083024 (2014).

[10] A.P. Smirnov and R.W. Harvey, Bull. Am. Physical Society 40, 1837 (1995).

[11] R. W. Harvey, and M. McCoy, in Proceedings of the IAEA Technical Committee Meeting on Simulation and Modeling of Thermonuclear Plasmas, Montreal, Canada, 1992 (IAEA, Vienna, 1992), US DOC NTIS Document No. DE93002962.

[12] M. Brambilla Nuc. Fusion 19, 1357 (1979).

[13] M. Brambilla Physics of Plasma Close to Thermonuclear Conditions (Brussels, 1980) 291.

[14] Y. Peysson and Tore Supra Team, Nuclear Fusion 41, 1703 (2001). doi:10.1088/0029-5515/41/11/320.

[15] N. J. Fisch, Phys. Rev. Lett. 41, 873 (1978). 
[16] A. Bécoulet, Ph. Bibet, F. Mirizzi, M.L. Apicella et al., Fus. Eng. Design 86, 490-496 (2011).

[17] S. Shiraiwa O. Meneghini, R.R. Parker, G. Wallace et al., Nucl. Fusion 51, 103024 (2011). 